\title{
Analysis of poultry eating and drinking behavior by software eYeNamic
}

\author{
A. De Montis, ${ }^{1}$ A. Pinna, ${ }^{1}$ M. Barra, ${ }^{1}$ E. Vranken ${ }^{2,3}$ \\ ${ }^{1}$ University of Sassari, Dipartimento di Agraria, Sassari, Italy; ${ }^{2}$ Fancom B.V., Panningen, The \\ Netherlands; ${ }^{3}$ KULeuven, Division M3-BIORES, Heverlee, Belgium
}

\begin{abstract}
Constant presence of at least one operator in livestock buildings for broilers would allow a perfect control of animal behaviour and, especially, deviations in feeding and drinking patterns, in the perspective of a high welfare status. However, as nowadays it is impossible for a farmer to be present in the farm all day long, automatic monitoring systems are required. The purpose of this paper is to introduce a system (eYeNamic) for automatic monitoring and analyzing broilers' behavior in a farm. eYeNamic is a camera system introduced and produced by Fancom BV, a company operating in the field of automation of livestock facilities. It includes three cameras located on the ridge of the broiler house and able to monitor chickens' behaviour twenty-four hours a day. Through eYeNamic it is possible to process the images and to obtain a measure of animals' distribution and activity, which can be conceived as valuable indicators of animal welfare. The study presented in this paper was divided into several phases: data collection, images visualization, observation of the distribution and activity of the chickens, and statistical analysis of the observations. The analysis of correlation between the number of 14 days old broilers near the feeding line (manual counted) and the average occupation density measured with eYenamic indicates that the best conditions have occurred with a $50 \mathrm{~cm}$ by $75 \mathrm{~cm}$ area around each feeding pan. With reference to the drinking line, the best response was found in an area $50 \mathrm{~cm}$ wide and the whole drinking line long. For the activity behavior, there was no significant correlation between activity and number of chickens eating from all the pans: this confirms that broilers while eating reduce their activity. It was concluded from this study that eYeNamic is a good system to observe animal behavior and, especially, to take care of their drinking and eating behaviour. A satisfactory correspondence between eYeNamic remote and human observations depends on a correct definition of animals' eating behaviour. In our case, this correspondence is established for the manual labeling, only if a broiler maintains its whole head inside the pan for a period lasting
\end{abstract}

Correspondence: Andrea De Montis, University of Sassari, Dipartimento di Agraria, viale Italia, 39, 07100 Sassari, Italy.

E-mail: andreadm@uniss.it

Key words: broilers, remote control, precision farming.

(C) Copyright A. De Montis et al., 2013

Licensee PAGEPress, Italy

Journal of Agricultural Engineering 2013; XLIV(s2):e33

doi:10.4081/jae.2013.s2.e33

This article is distributed under the terms of the Creative Commons Attribution Noncommercial License (by-nc 3.0) which permits any noncommercial use, distribution, and reproduction in any medium, provided the original author(s) and source are credited.
20 seconds. In many cases the simple closeness to the pan or drinking line does not guarantee that a broiler is eating or drinking.

\section{Introduction}

Nowadays, animal welfare is an important public concern and unlimited access to food and water are important parameters in relation to welfare. These conditions result in improved animal performance, stronger growth, less illness, and lower mortality rates.

Hence, continuous observation of animal performance is very important because it is impossible for farmers to be present in the animal houses the whole day. Since today there is lack of systems able to autonomously observe specific animals, such as broilers, Fancom BV, a company operating in the field of automation of livestock facilities, has introduced the system eYeNamic. The experimentation of eYeNamic has to be referred to a panorama of research studies, which recently have focused on the requirements for remote and continuous livestock monitoring systems. The purpose of this paper is to introduce a system for automatic monitoring and analyzing broilers' behavior in a farm located in the Limburg province, The Netherlands and to demonstrate its usefulness in detecting and correcting unusual or inconvenient animals' behaviour. In the next section, a brief literature review on animals' remote monitoring systems is presented. In the third section, the monitoring system selected for the experimentation is introduced and commented. In the fourth section, the results of this work are discussed, while in the fifth section the conclusions are proposed.

\section{Animals' remote monitoring systems: a brief literature review}

According to Laurence (2008), applied behavioural scientists will focus not just on animal behaviour per se, but specifically on the resolution of key issues about animal welfare. He observes that today public concern about welfare in livestocks and, generally, quality of animal life is still expanding being comparable to other conteporary issues, such as food supply. A particular interest is devoted to the development of experimental modelling able to offer proper welfare assessment measures. In this respect, research studies have focussed on the integration of a number of livestock monitoring systems through software and hardware able to gather and organize a wealth of information. Technological advances include sensors management and image capture and analysis capable of control key variables, such as animal identity, weight, and behaviour, physiological and environmental factors, and body conformation and composition, and odours and sounds (Frost et al., 1997).

The panorama of studies on livestock monitoring systems and animal behaviour embraces works on a number of species including broiler chickens. We report some examples as follows.

Dawkins et at (2012) study broilers' behaviour by means of camera equipment and statistical analysis of optical flow patterns and found that collective movements of the flock are significantly correlated with 
relevant welfare measures, i.e. mortality, hockburn, and abnormal walking. Kristensen and Cornou (2011) focus on deviations from normal activity level of broiler chickens behaviour. They experimented with an overhead video camera system coupled with an outliers filtering software based on linear dynamic modeling. One of the main findings of these authors is the test of a device able to automatically detect abnormal activity levels and emit an alarm to immediately notify producers. Aydin et al. (2010) start from the evidence that metabolic and locomotive problems are related to fast growth rate and inactivity. Thus they study the activity level of broiler chickens by means of camera recording and processing software EyeNamic (Leroy et al., 2006) and found high correlation between gait score and activity level.

As this paper refers to the aforementioned software, in the next section we present the features of this monitoring system and the experimentation developed.

\section{Materials and methods}

EYeNamic is a camera system used to monitor the behaviour of animals. There are three cameras mounted in the ridge of the chicken house that steadily monitor the floor, so you can analyze the behaviour of broilers and follow it from minute to minute. A scheme explaining the detail of cameras' location is reported in Figure 1. Afterwards, analysis software translates these images into an index for animal distribution and activity in the house, both valuable indicators of animal welfare. EYeNamic software allows to:

-- Measure the distribution and activity level of animals (broiler chickens) in real time.

- Localize the occupation density and activity by measuring it for different pre-defined zones.

- Freely choose the number and placement of the zones using a configuration tool.

The occupation density is the percentage of pixels representing chickens in the pictures in relation to the total amount of pixels in one zone: therefore, it is obtained by calculating the ratio between the number of object pixels over total pixels. A uniformity index was defined in order to know which percentage of occupation density values fall within the $(+/-20 \%)$ range around the average occupation density. A scheme of the processing rationale is reported in Figure 2.

The average activity index is a measure of the animals' dynamics and is calculated by processing the data of the activity density index. The activity density index is calculated by confronting pictures taken by the same camera during a time period equal to 1 second. A scheme of
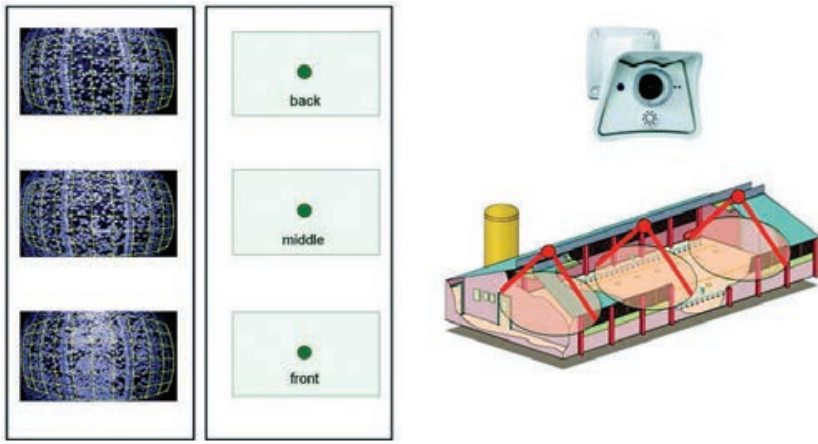

Figure 1. eYeNamic positioning and perspective of the three cameras. the processing rationale is reported in Figure 3.

As the scheme reported in Figure 4 shows, the system includes a monitoring and a processing module: livestock floor pictures are taken every second by the three cameras, sent continuously in a storing device and analyzed through the software eYeNamic in order to obtain automatically a characterization of animals' activity and distribution. In addition, the number of eating and drinking chickens is manually
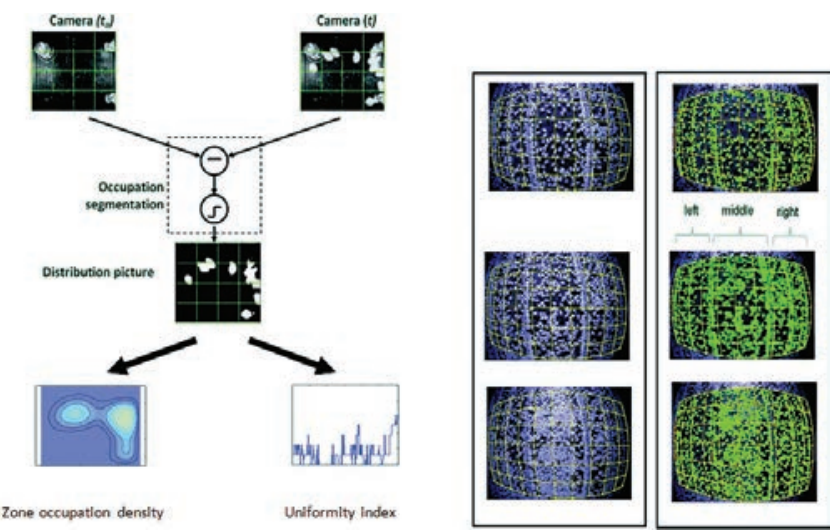

Figure 2. eYeNamic scheme of calculation for chickens' occupation index.

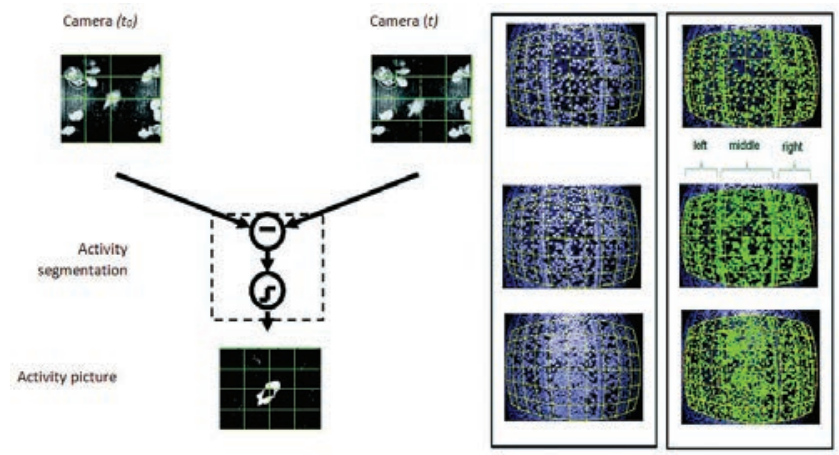

Figure 3. eYeNamic scheme of calculation for chickens' activity index.

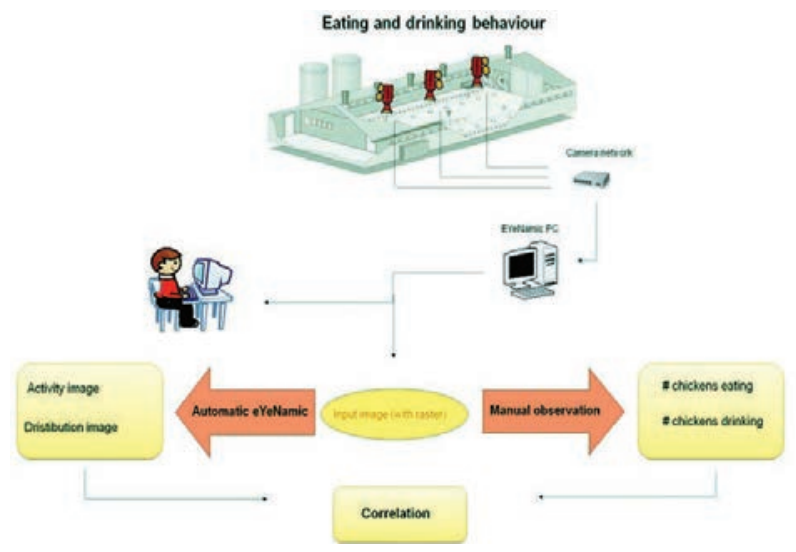

Figure 4. Scheme of processing and comparing automatically versus manually retrieved information. 
calculated and confronted with the automatically processed figures obtained by means of correlation analysis.

The images have been collected each 7th day of the week, during the period from July 15 to August 19, 2010, and light period from 3:00:00 AM to 7:00:00 AM. The manually count of eating and drinking chickens has been executed on pictures shot every five minutes. Hence, information from eYeNamic processing and manual observation has been synchronized in a compact dataset covering 42 days. For the manual labeling, only the chickens with their whole head inside the pan in the period from 10 seconds before until 10 seconds after the picture were counted at four feeding pans in the camera image. In Figure 5, the localization of the feeding pan is reported. These data were compared with the results of eYeNamic in which we selected the data of the occupation density in the zones around the feeding pans.

Different combinations of zones were used for the automatic analyses (see figures $6 ; 7 ; 8 ; 9$; and 10). In total, we have considered five different configurations for the whole chickens growth cycle; afterwards, we defined the optimal area in the pictures occupied by chickens eating at the feeding pan. Then we checked the best configuration of eating zones by comparing data from eYeNamic with the data of the manual observations.

A similar procedure was used also to calculate the average density for drinking lines: drinking pattern with eYeNamic data have been compared with numbers of drinking animals manually observed (see Figure 11). Different configurations of zones were used also for this analysis.

In this way, it is possible to assess the effectiveness of eYeNamic as an animals' behavior automatic detection system. In the next section, the results of the behavior analysis is presented and referred to 14 days old chickens.

\section{Results}

We develop a correlation analysis to inspect the interaction between the number of eating chickens and average occupation density of a specific zone around each pan for five configurations. The analysis allows to see the best configuration around each pan. In Table 1, we report the correlation coefficients.

We can infer that for each pan the highest correlation coefficient corresponds to configuration 3 (average value 0.66 ), even though high correlation coefficients occur for some other pans; (see, for example, pan three, configuration 4, and 5). So, we can confirm that the highest concentration of chickens eating around the pans occurs on average for configuration 3 . By contrast, in configuration 1 the average correlation coefficient is the lowest ( 0.53$)$ : this means that there are more chick-

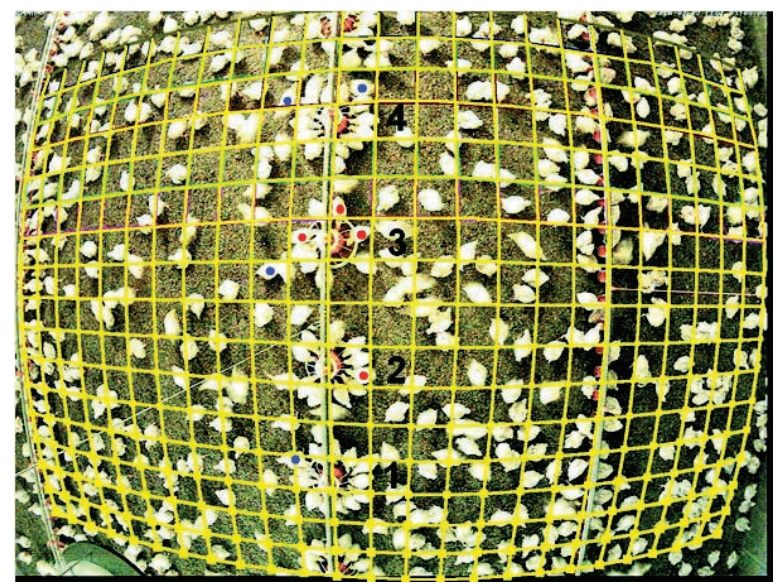

Figure 5. Chickens' feeding behavior around the pans: red points correspond to eating and blue points to not eating animals.

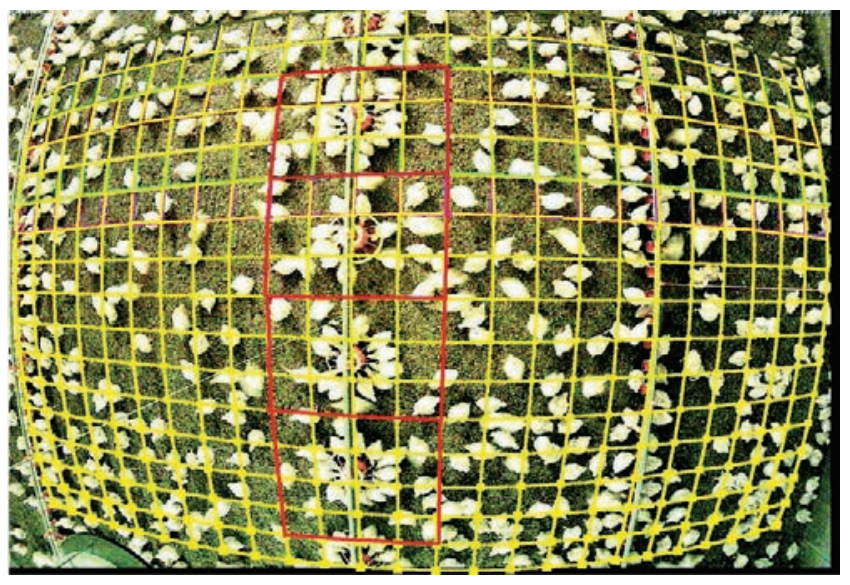

Figure 6. Areas around the pans adopted as reference zones to calculate the average density for configuration 1 .

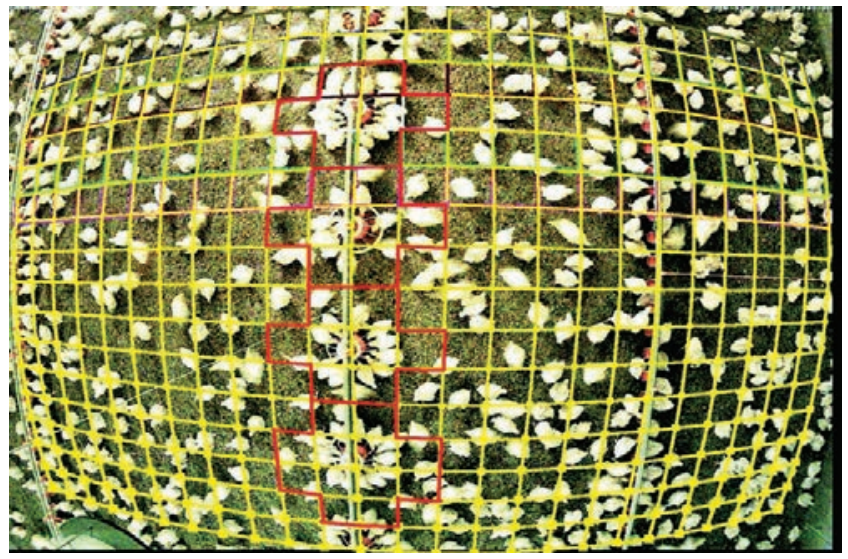

Figure 7. Areas around the pans adopted as reference zones to calculate the average density for configuration 2 .

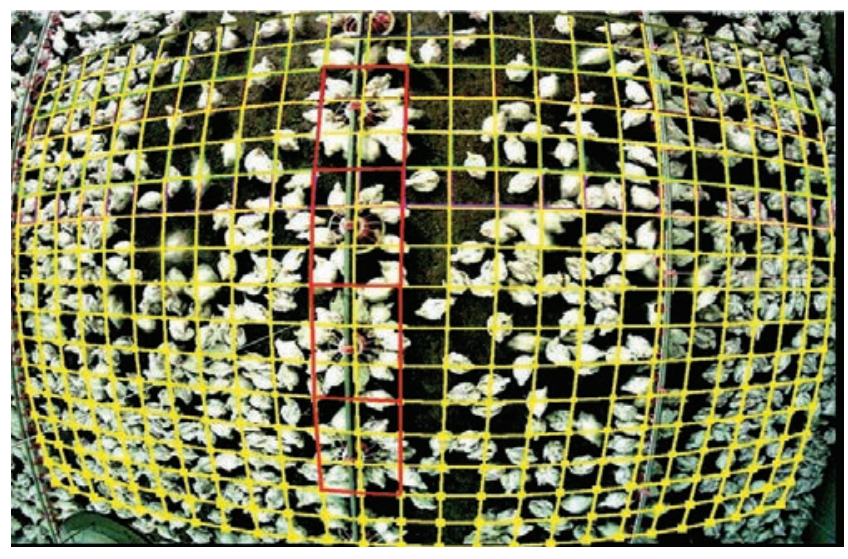

Figure 8. Areas around the pans adopted as reference zones to calculate the average density for configuration 3 . 
Table 1. Correlation analysis of average occupation density versus eating pattern and average activity density versus eating pattern.

\begin{tabular}{|c|c|c|c|c|}
\hline \multirow[t]{2}{*}{ Feeding pan } & \multirow[t]{2}{*}{ Configuration } & \multicolumn{3}{|c|}{ Correlation coefficient (R) } \\
\hline & & Average occupation density vs eating pattern & & Average activity vs eating pattern \\
\hline \multirow[t]{5}{*}{1} & 1 & 0.55 & & 0.48 \\
\hline & 2 & 0.60 & & 0.52 \\
\hline & 3 & 0.73 & & 0.27 \\
\hline & 4 & 0.63 & & 0.24 \\
\hline & 5 & 0.55 & & 0.37 \\
\hline \multirow[t]{5}{*}{2} & 1 & 0.63 & & 0.30 \\
\hline & 2 & 0.59 & & 0.35 \\
\hline & 3 & 0.69 & 0.18 & \\
\hline & 4 & 0.71 & 0.19 & \\
\hline & 5 & 0.51 & 0.14 & \\
\hline \multirow[t]{5}{*}{3} & 1 & 0.54 & 0.22 & \\
\hline & 2 & 0.63 & 0.14 & \\
\hline & 3 & 0.68 & 0.00 & \\
\hline & 4 & 0.72 & 0.04 & \\
\hline & 5 & 0.72 & 0.04 & \\
\hline \multirow[t]{5}{*}{4} & 1 & 0.42 & 0.23 & \\
\hline & 2 & 0.52 & 0.13 & \\
\hline & 3 & 0.53 & 0.16 & \\
\hline & 4 & 0.47 & 0.13 & \\
\hline & 5 & 0.57 & 0.10 & \\
\hline
\end{tabular}

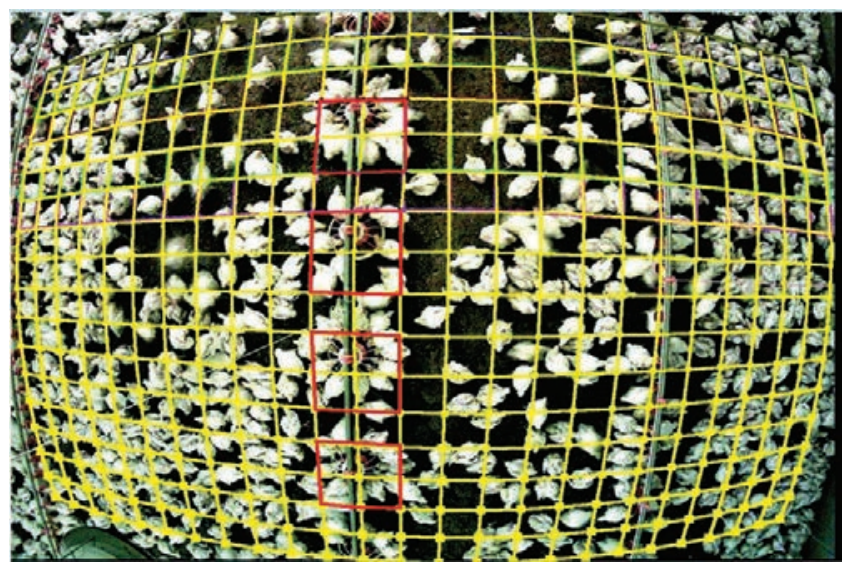

Figure 9. Areas around the pans adopted as reference zones to calculate the average density for configuration 4 .

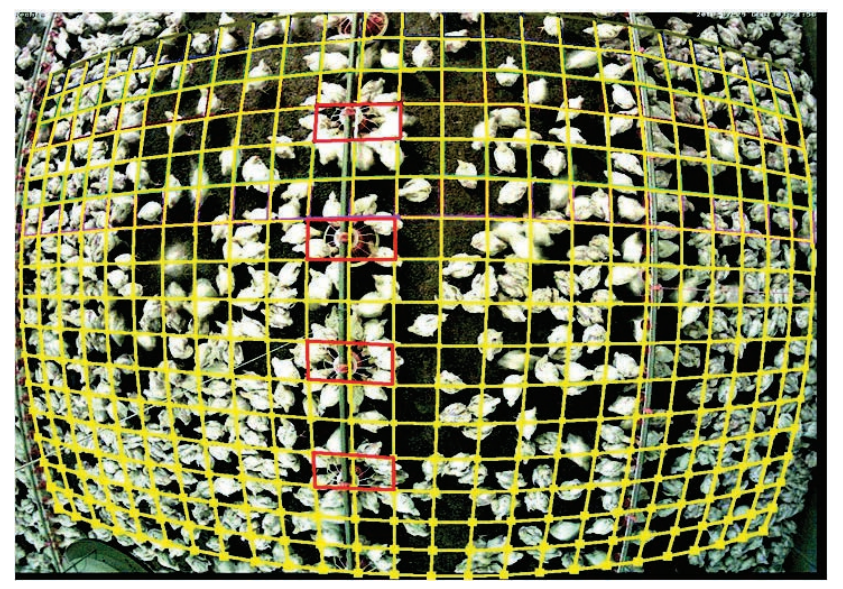

Figure 10. Areas around the pans adopted as reference zones to calculate the average density for configuration 5 .

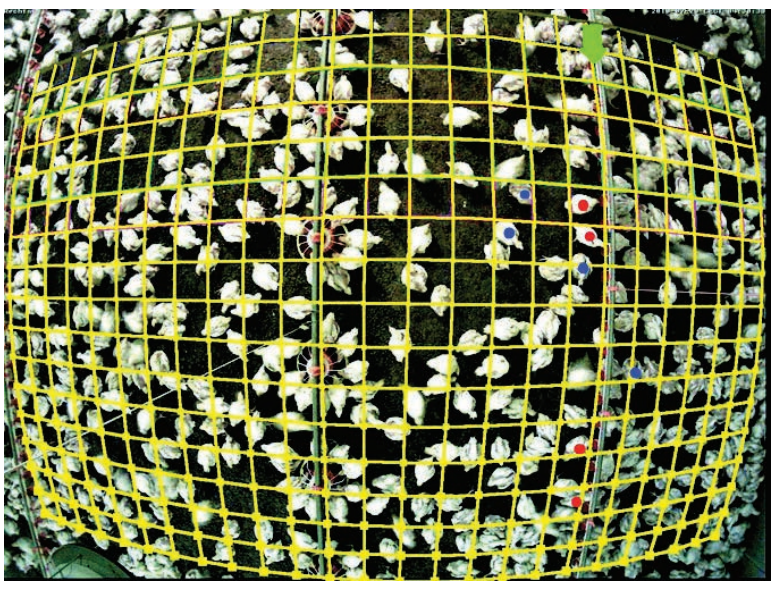

Figure 11. Analysis of drinking behavior: the green arrow indicates the drinking line; red points identify drinking and blue point not drinking chickens.

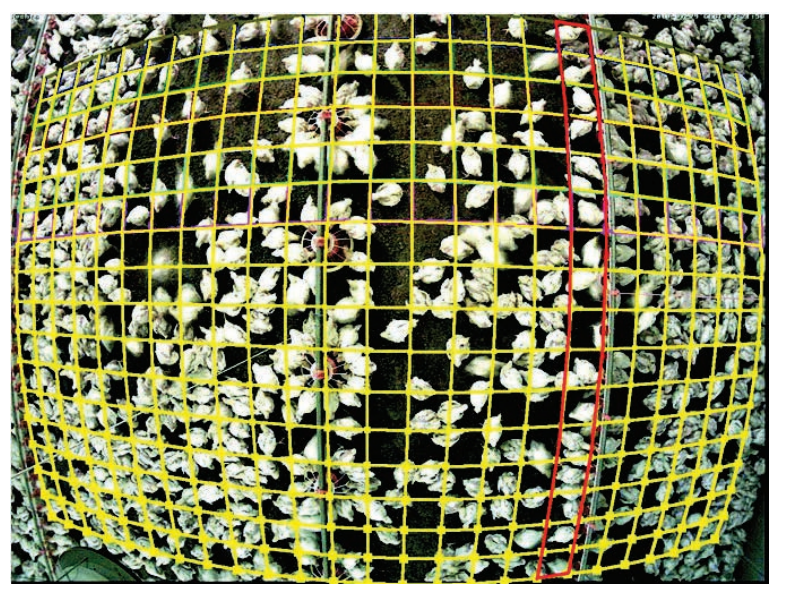

Figure 12. Area around the drinking line adopted as reference zone to calculate the average density for configuration $\mathrm{A}$. 


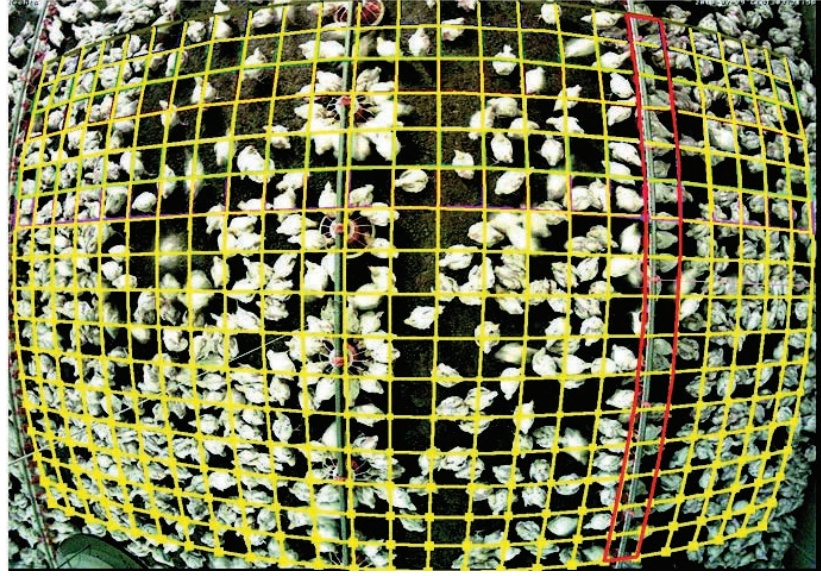

Figure 13. Area around the drinking line adopted as reference zone to calculate the average density for configuration $B$.

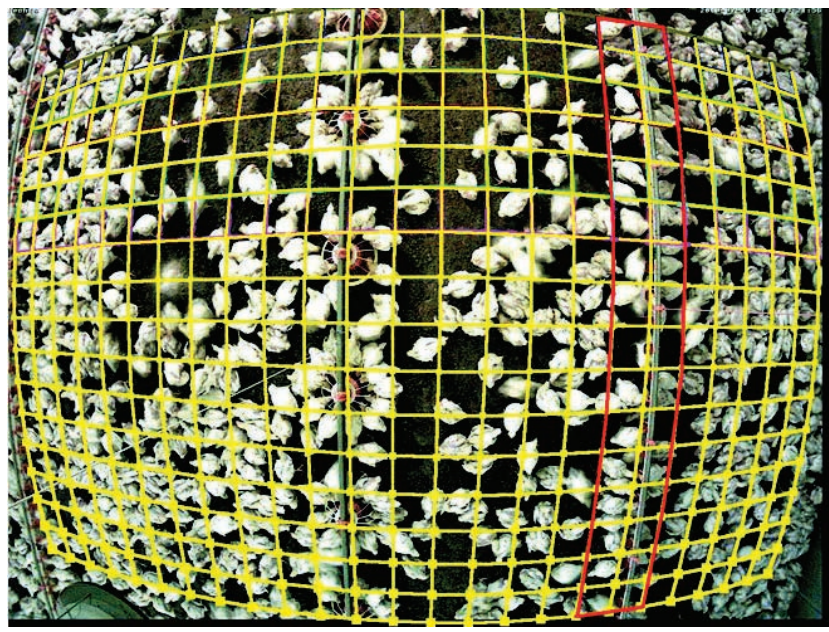

Figure 14. Area around the drinking line adopted as reference zone to calculate the average density for configuration $\mathrm{C}$.

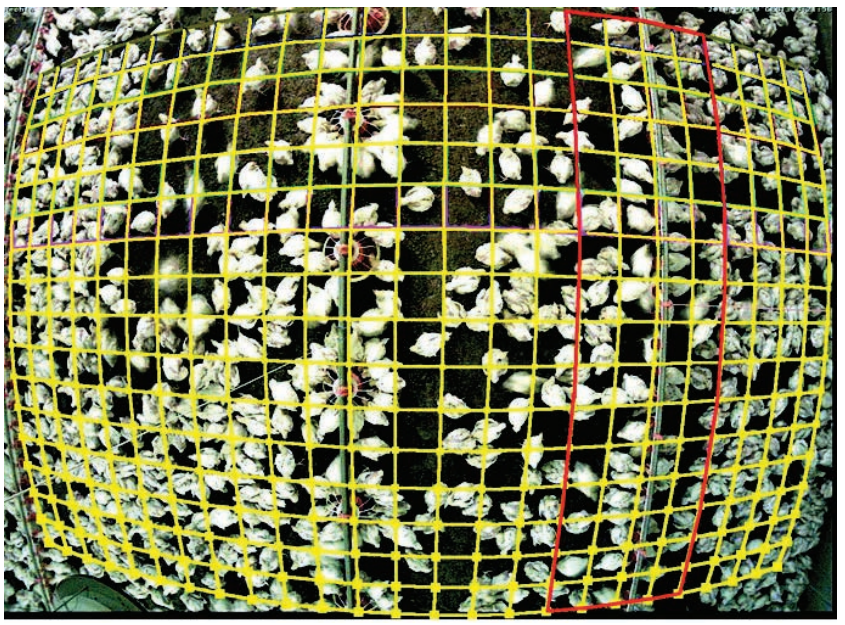

Figure 15. Area around the drinking line adopted as reference zone to calculate the average density for configuration $\mathrm{D}$.

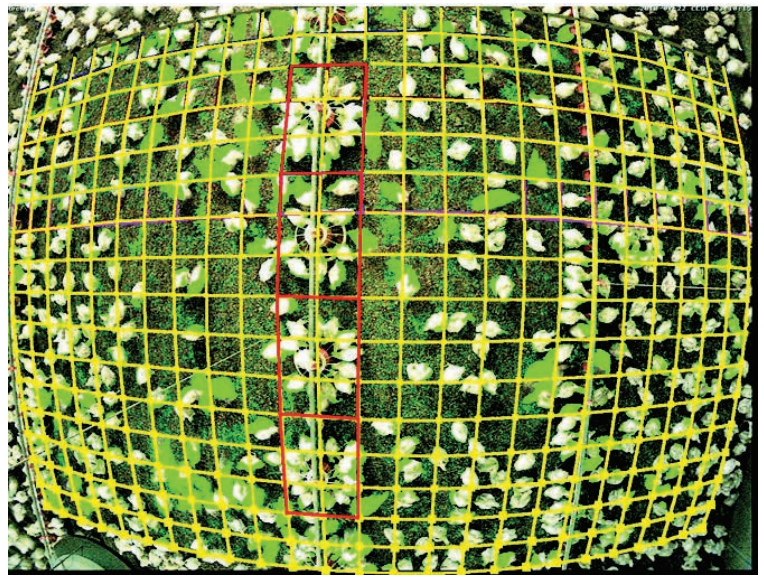

Figure 16. Analysis of chickens' movements: the green arrows describe the activity for configuration 3 .
Figure 17. Analysis of chickens' movements: the green arrows describe the activity for configuration 1 .

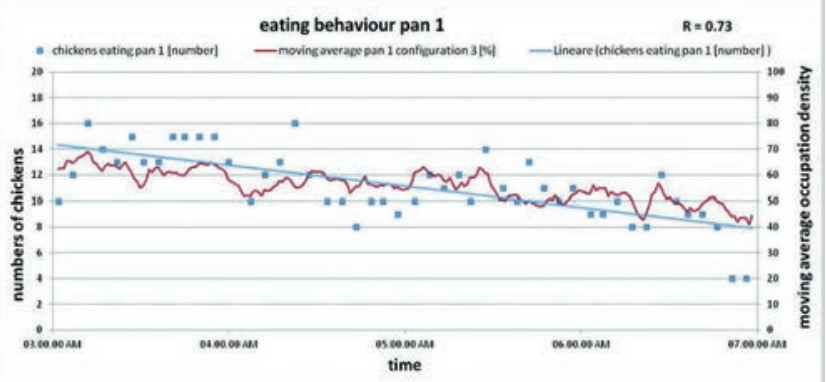

Figure 18 Functional relation between number of chickens eating at pan 1 and average occupation density for configuration 3 . 
ens that are not eating in the feeding zones in configuration 1 than in configuration 3 .

Table 1 also reports correlation coefficients relating number of eating chickens and average activity for each pan and for five configurations. Results show that for configuration 3 a much lower correlation coefficient (average 0.15 ) is found compared to other configurations. This implies clearly that birds do not move, while eating, i.e. their level of activity is usually low. The high correlation coefficient for configuration 1 means that eating chickens have been moving with a more intense activity.

Figures 16 and 17 report the same image but with two different configurations; it is possible to monitor the activity of the chickens and also select a number of not eating animals.

In Figures 18 and 19, we report graphs about the functional relation between the numbers of eating chickens at pans 1 and 4 and the average occupation density for configuration 3 . The horizontal axis indicates a time period from 3:00:00 AM to 7:00:00 AM, while the primary vertical axis indicates the number of eating chickens manually counted every five minutes. The second vertical axis shows the moving average occupation density. It is possible to see a better correlation for pan 1 through the correlation coefficient.

The number of chickens eating, like the moving average occupation density, decreases with time from 3:00:00 AM to 7:00:00. It is possible to see that they are concentrated especially in the first hour of the light period.

A similar work was done for inspecting the drinking behavior. We calculated the correlation coefficient for the whole drinking line for four configurations: in this way it is possible to see the best configuration of the zones around the drinking line. Correlation analysis results are reported in Table 2 .

We found the highest correlation coefficient $(0.65)$ for configurations $\mathrm{C}$ : this implies that most of the chickens around the drinking line with combination $\mathrm{C}$ are drinking. By contrast, for configuration $\mathrm{D}$ the correlation coefficient is not significant: hence in this case, there are a lot of chickens not drinking.

Figure 20 reports on the functional relation between the number of (manually counted) drinking chickens, and the average occupation density for combination $\mathrm{C}$. The horizontal axis indicates the time period of the observation, the left hand side vertical axis indicates the number of drinking chickens, and the right hand side vertical axis indicates the moving average occupation density during the 4 hours. The number of drinking chickens decreases very slightly with time. By contrast, the average occupation density is constant over time: there are a lot of chickens that are not drinking in that area.

Figure 21 report a graph concerning chickens' eating behavior during the whole growth cycle. The highest correlation coefficients occur for 2 weeks old chickens for all configurations. Moreover, configuration 3 is the best for 7 to 42 days old chickens. For 5 and 6 weeks old chickens correlation coefficient is lower than for 14 days old animals: this phenomenon occurs since in the last 2 weeks there are less eating chickens in a larger surface, as some animals are not eating, while moving around the pan. It is important to see that with all the configurations for one week old chickens correlation coefficient increases in the period from 15/07/2010 until to 22/07/2010 and over time also the number of eating chickens increases. Afterwards, correlation coefficient is always lower in each configuration.

\section{Conclusions}

In this section, we discuss the results of this paper. We have presented a remote monitoring system able to control chickens' behaviour by
Table 2 Correlation analysis of average occupation density versus drinking pattern.

Drinking line configuration_ Correlation coefficient (R)

\begin{tabular}{ll} 
A & 0.60 \\
B & 0.45 \\
\hline C & 0.65 \\
D & 0.43 \\
\hline
\end{tabular}

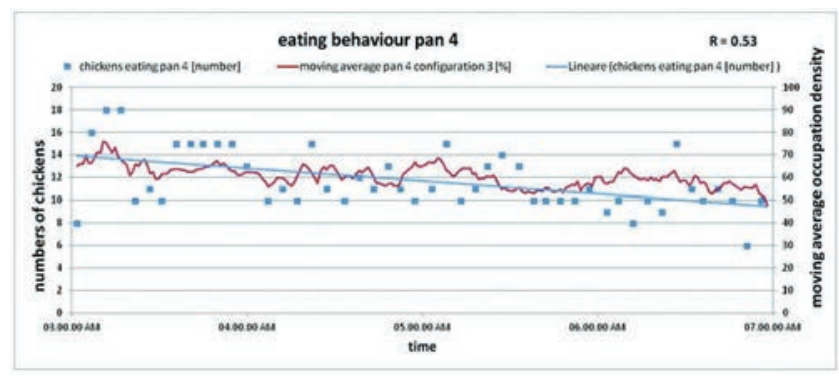

Figure 19 Functional relation between number of chickens eating at pan 4 and average occupation density for configuration 3 .

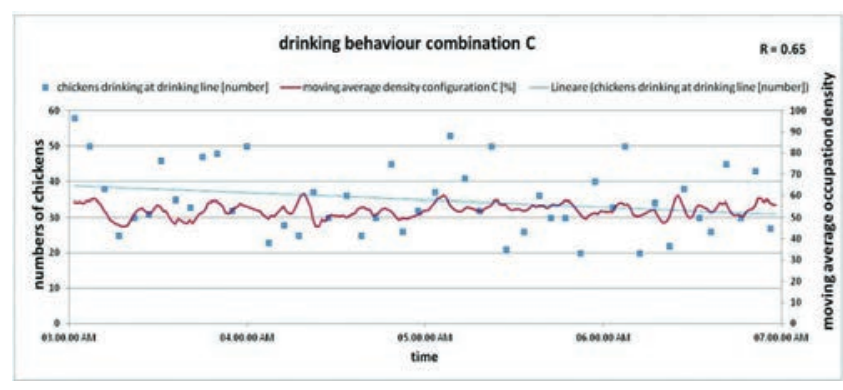

Figure 20 Functional relation between number of drinking chickens and average occupation density for configuration $\mathrm{C}$.

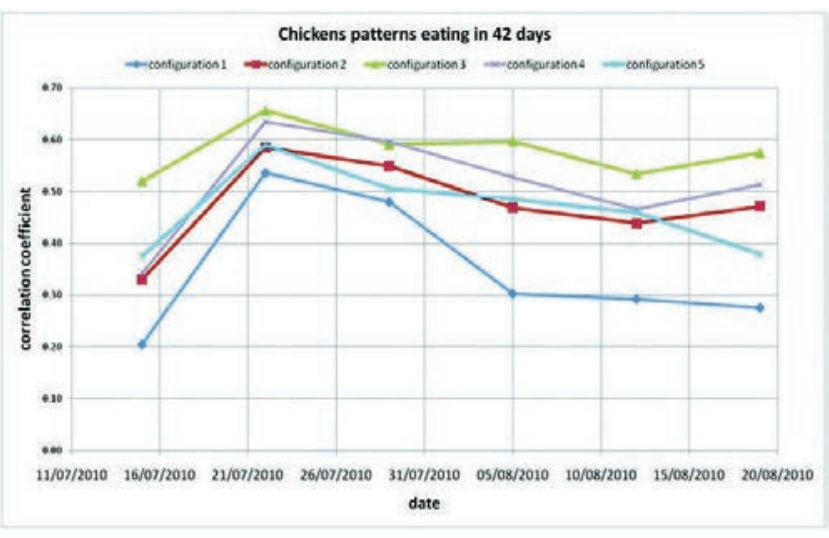

Figure 21 Chickens' eating behavior by configuration during the whole growth cycle. 
processing images taken from three fixed cameras. We found that for eating behaviour there is a good correlation between the number of eating chickens and average occupation density for 14 days old chickens. The highest correlation coefficient (average value equal to 0.66 ) was found at pan 4 for configuration 3 referring to an area extending $50 * 75 \mathrm{~cm}$ around the feeding line. For drinking behavior, there is a good correlation coefficient for configuration $\mathrm{C}(0.65)$ corresponding to a zone $50 \mathrm{~cm}$ wide around the drinking line. For the activity behaviour there is no significant correlation for all the pans, and especially for configuration 3 . In fact, for this configuration there is a low correlation coefficient for the activity (0.15), in contractition to the highest correlation for occupation density.

As a conclusion of these analyses, we can say that eYeNamic is a good system to observe animal behaviour, especially to take care of the animals' welfare. However, it is necessary to find a good definition of eating and drinking chickens to improve the correspondence between data processed through eYeNamic system and the real manually counted values. Moreover, with an overview of that relationship we can say that it is very important to analyze the areas close to the feeding pan and drinking line. That because usually with a bigger raster we observe a number of birds in the specific zones that are not eating or drinking.

\section{References}

Aydin A., Cangar 0., Eren Ozcan S., Bahr C., Berckmans D. 2010. Application of a fully automatic analysis tool to assess the activity of broiler chickens with different gait scores. Computers and Electronics in Agriculture 73: 194-199.

Dawkins M.S., Cain R., Roberts S.J. 2012. Optical flow, flock behaviour and chicken welfare. Animal Behaviour 84: 219-223.

Frost A.R., Schofield C.P., Beaulah S.A., Mottram T.T., Lines J.A., Wathes C.M. 1997. A review of livestock monitoring and the need for integrated systems. Computers and Electronics in Agriculture 17: 139159.

Kristensen H.H., Cornou C. 2011. Automatic detection of deviations in activity levels in groups of broiler chickens - A pilot study. Biosystems Engineering 109: 369-376.

Lawrence A.B. 2008. Applied animal behaviour science: Past, present and future prospects. Applied Animal Behaviour Science 115: 1-24.

Leroy T., Vranken E., Van Brecht A., Struelens E., Sonck B., Berckmans D. 2006. A computer vision method for on-line behavioral quantification of individually caged poultry. Transaction of ASABE 49 : 795 802. 